Revista de Investigación de Física. Vol. $12 \mathrm{~N}^{\circ} 1$ (2009) 05-13

\title{
CARACTERIZACIÓN MINERALÓGICA DE UN IMPACTO METEORITICO EN LA LOCALIDAD DE CARANCAS -PUNO
}

\author{
María L. Cerón Loayza ${ }^{\mathrm{a}^{*}}$, Jorge A Bravo Cabrejos ${ }^{\mathrm{a}}$ \\ ${ }^{a}$ Laboratorio de Análisis de Suelo, Facultad de Ciencias Físicas, Universidad Nacional Mayor de San Marcos Ap. Postal \\ 14-0149, Lima 14, Perú.
}

(Recibido Junio 01, 2009; Aceptado Setiembre 06, 2009)

\begin{abstract}
Resumen
Reportamos los resultados del estudio del meteorito caído en una zona deshabitada vecina al pueblo de Carancas, región Puno, a unos 1,300 km al sur de Lima. Los estudios realizados por difractometría de rayos X, espectroscopia Mössbauer de transmisión (tomados a temperatura ambiente y a 4,2 K), y por fluorescencia de rayos $\mathrm{X}$ de energía dispersiva revelan la presencia en la muestra del meteorito de sitios magnéticos adjudicados a las fases de $\mathrm{Fe}-\mathrm{Ni}$ y a la troilita $(\mathrm{Fe}, \mathrm{S})$, y de 3 dobletes paramagnéticos, dos de ellos asignados al $\mathrm{Fe}^{2+}$, uno asociado al olivino y el otro al piroxeno, y el tercero debido a un sitio ocupado por $\mathrm{Fe}^{3+}$, el cual puede estar asociado a óxidos súper paramagnéticos y/o a hidróxidos de Fe. Las muestras del suelo del cráter revelan una composición que consiste principalmente de cuarzo, albita e impactitas como coesita y stishovita $\left(\mathrm{SiO}_{2}\right)$. La presencia de estas fases con alta presencia de $\mathrm{SiO}_{2}$ en los suelos del cráter fortalece la hipótesis de su origen por metamorfismo inducido por impacto; también observamos la presencia del óxido de Fe hematita, de alúmino silicatos como illita y montmorillonita, y una fase de $\mathrm{Fe}^{3+}$ por asignar. En términos generales los resultados obtenidos con estas técnicas se complementan bastante bien y permiten verificar el origen de las muestras analizadas.
\end{abstract}

PACS: 33.45.+x; 61.05.C-; 78.70.En

Palabras claves: Espectroscopia Mössbauer, difractometría de rayos x, fluorescencia de rayos x, meteorito, Carancas.

\begin{abstract}
We report the results of the study of a meteorite that impacted an inhabited zone in the neighborhood of the town of Carancas, Puno Region, about $1,300 \mathrm{~km}$ south of Lima. The analysis carried out by $\mathrm{X}$ ray diffractometry, transmission Mössbauer spectroscopy (at room temperature and at 4,2 K), and by energy dispersive $\mathrm{X}$ ray fluorescence reveal the presence in the meteorite simple of magnetic sites assigned to the $\mathrm{Fe}-\mathrm{Ni}$ and troilite $(\mathrm{Fe}, \mathrm{S})$ phases, and of 3 paramagnetic doublets, two of them assigned to $\mathrm{Fe}^{2+}$, one associated to olivine and the other to pyroxene, and the third one due to a site occupied by $\mathrm{Fe}^{3+}$, which can be associated to oxides in a superparamagnetic state and/or by an $\mathrm{Fe}$ hydroxide. The soil samples from the crater reveal a composition that consists mainly of quartz, albite and impactites such as coesite and stishovite $\left(\mathrm{SiO}_{2}\right)$. The occurrence of these phases with a high content of $\mathrm{SiO}_{2}$ in the crater soils strengthens the hypothesis of their origin induced by impact; we observe also the presence of the Fe oxide hematite, of aluminum silicates such as illite and montmorillonite, and an unasigned phase of $\mathrm{Fe}^{3+}$. In general, the results obtained by these techniques complement each other rather well and allow the verification of the origin of the studied simples.
\end{abstract}

Keywords: X ray fluorescence, X ray diffractometry, transmission Mössbauer spectroscopy, meteorite, Carancas.

\section{Introducción}

El día 15 de setiembre del año 2007 se reportó el impacto de un meteorito en una zona vecina al pueblo de Carancas, distrito de Desaguadero, provincia de Chucuito, región Puno, cerca de la frontera con Bolivia. Este impacto causó una gran explosión y la formación de un cráter de unos $20 \mathrm{~m}$ de diámetro y algo más de $3 \mathrm{~m}$ de profundidad. También se reportó el desprendimiento de gases sulfurosos que irritaron la vista y fosas nasales de los vecinos del lugar. Por informaciones periodísticas se decía que el meteorito era una muestra de material radiactivo, motivo por el cual se llevó un contador Geiger modelo Radalert 50, el cual dio resultados negativos, dando valores de $0,030 \mu \mathrm{S}$. En el lugar del

\footnotetext{
*Correspondig author.e-mail: malucelo@hotmail.com
} 
impacto la capa freática yace a unos $3 \mathrm{~m}$ de profundidad, por lo que este cráter yace en la actualidad lleno de agua. La foto que se muestra en la Figura $\mathrm{N}^{\circ} 1$ fue tomada 5 días después del impacto.

\section{Materiales y Metodos}

La recolección de las muestras se realizó 5 días después de ocurrido el impacto en el sector de Carancas. La muestra de meteorito es de color gris y de textura fina, con pequeñas estrías en su superficie, que podrían deberse al impacto con el suelo.

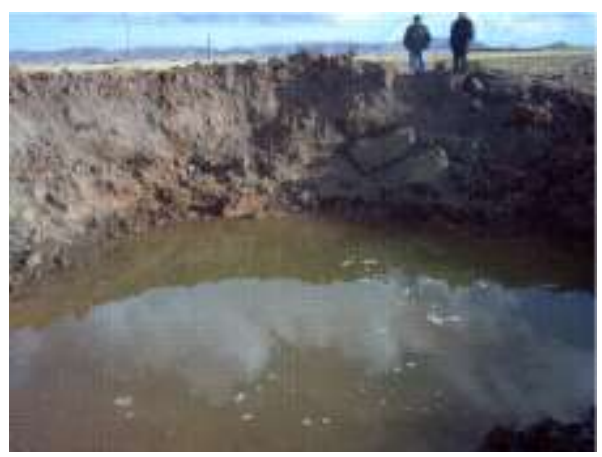

Fig. 1. Lugar de impacto del meteorito de Carancas- Puno.

La preparación del análisis del meteorito se hizo con los residuos que se desprendieron del total de la muestra, logrando obtener así una masa de $180 \mathrm{mg}$ de muestra, necesaria y suficiente para el análisis por espectroscopia Mössbauer de transmisión (EMT). Previo a su colocación en una porta muestra, este material fue molido en un mortero de ágata y pasada por un tamiz de 160 micras.

Las muestras del suelo del cráter se obtuvieron con la colaboración del Dr. J. Ishitsuka (Instituto Geofísico de Perú). Éstas también fueron tamizadas y molidas en un mortero de ágata, tomando para su análisis aprox. $200 \mathrm{mg}$, y se las designó como:

M1: Muestra del cráter / parte interna del borde, de color rojizo.

M2: Muestra del cráter/ parte sobre el borde, de color gris.

M3: Muestra del cráter/ parte externa del cráter, de color marrón.

El análisis de la composición elemental se realizó empleando un equipo portátil de fluorescencia de rayos X (FRX) marca Amptek que utiliza un tubo de rayos $\mathrm{X}$ con blanco de $\mathrm{Ag}$, que opera a $30 \mathrm{kV}$ y 50 $\mu \mathrm{A}$, ver Fig. 2. Esta técnica permite reconocer elementos químicos de $\mathrm{Z}>12$ (magnesio) En la muestra de meteorito (ver Fig. 3(a)), se observa la presencia de los elementos que se listan en la Tabla 1, que nos muestra de manera particular la alta concentración de hierro, así como de azufre, cloro, cromo y níquel en mayores proporciones que en las muestras del suelo del cráter. La incertidumbre en la medida de las concentraciones es del orden de $10 \%$ aproximadamente. También podemos observar en la Fig. 3(c) el espectro simulado por un programa para análisis cuantitativo. La presencia de silicio se da en menor concentración que en un suelo

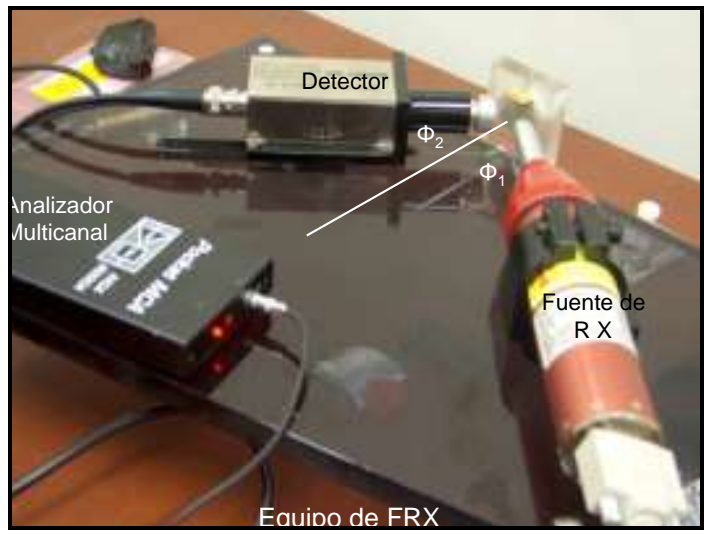

Fig. 2. Equipo de fluorescencia de rayos X- UNMSM, Facultad de Ciencias Físicas

Tabla 1 Análisis cuantitativo de las muestras del meteorito de Carancas- Puno, y de las muestras M1, M2 y M3 del suelo del cráter.

\begin{tabular}{ccccc}
\hline & \multicolumn{4}{c}{ Muestras } \\
\hline Elementos & Meteorito & $\begin{array}{c}\text { M1 } \\
(\%)\end{array}$ & $\begin{array}{c}\text { M2 } \\
(\boldsymbol{\%})\end{array}$ & $\begin{array}{c}\text { M3 } \\
(\boldsymbol{\%})\end{array}$ \\
\hline $\mathrm{Al}$ & 7.5 & 9.0 & 9,8 & 9,7 \\
$\mathrm{Si}$ & 15.0 & 27.0 & 21,2 & 24.0 \\
$\mathrm{~S}$ & 0.80 & 0,012 & 0,013 & 0,013 \\
$\mathrm{Cl}$ & 0.80 & 0,70 & 0,47 & 0,76 \\
$\mathrm{~K}$ & 0.28 & 1,43 & 1,36 & 1,05 \\
$\mathrm{Ca}$ & 0.66 & 0,92 & 1,37 & 3,46 \\
$\mathrm{Ti}$ & 0.080 & 0,38 & 0,30 & 0,37 \\
$\mathrm{Cr}$ & 0.11 & 0,090 & 0,010 & 0,010 \\
$\mathrm{Mn}$ & 0.12 & 0,104 & 0,074 & 0,122 \\
$\mathrm{Fe}$ & 5.78 & 3,39 & 1,69 & 3,30 \\
$\mathrm{Ni}$ & 0.20 & 0,007 & 0,003 & 0,007 \\
$\mathrm{Cu}$ & -- & 0,058 & 0,043 & 0,060 \\
$\mathrm{Zn}$ & 0.005 & 0,071 & 0,059 & 0,076 \\
$\mathrm{La}$ & 0.10 & -- & -- & -- \\
$\mathrm{Rb}$ & -- & 0,010 & 0,006 & 0,010 \\
$\mathrm{Sr}$ & -- & 0,011 & 0,053 & 0.022 \\
\hline
\end{tabular}

Para la identificación estructural de los compuestos presentes en las muestras se recurrió a la técnica de difractometría de rayos X (DRX), usando un difractómetro marca RIGAKU modelo MINIFLEX, el cual usa una radiación de $\mathrm{Cu}-\mathrm{K} \alpha$ con $\lambda=1,54178 \AA$, con un goniómetro vertical. El ángulo de barrido fue de $4^{\circ}<2 \theta<70^{\circ}$ y el avance en $2 \theta$ fue 
de $0,02 \%$ paso con un intervalo de 3 segundos por paso.

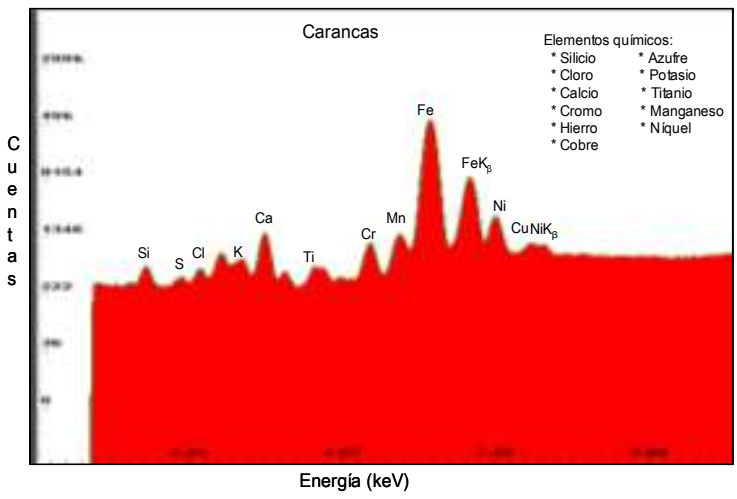

(a)

Fig. 3(a). Espectros de FRX de la muestra del meteorito de Carancas- Puno

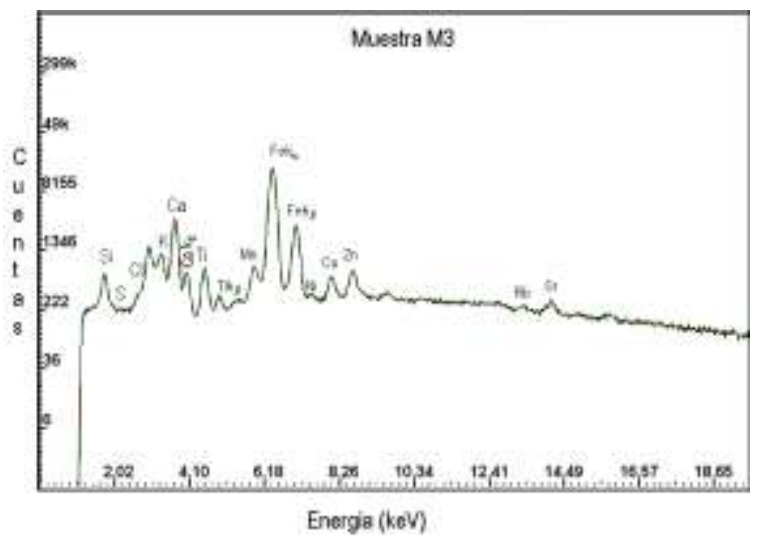

(b)

Fig. 3(b). Espectros de FRX de la muestra M3 del suelo del cráter.

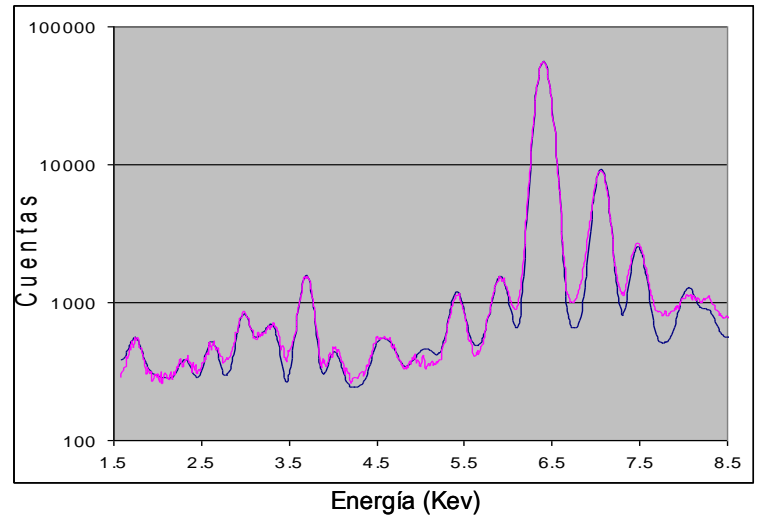

(c)

Fig. 3(c). Espectros de FRX de la muestra del meteorito conjuntamente con su espectro simulado (Bravo C., J. A. (2009). comunicación privada).

Para obtener información más detallada sobre los compuestos que contienen Fe se recurrió a la técnica de espectroscopia Mössbauer de transmisión (EMT). Para este fin se utilizó un equipo convencional operado a velocidad sinusoidal y 1024 canales. Los espectros Mössbauer de todas las muestras fueron tomados a temperatura ambiente (TA) en el Laboratorio de Arqueometría - Facultad de Ciencias Físicas - UNMSM; en el caso de la muestra del meteorito, se tomó espectros a temperatura de helio líquido (THL) en colaboración con el Centro Brasileiro de Pesquisas Físicas (CBPF). Los espectros se tomaron en geometría de transmisión usando una fuente de ${ }^{57} \mathrm{Co}$ en matriz de $\mathrm{Rh}$ y fueron analizados usando el programa Normos de Brand [1] en la versión de sitios cristalinos (Normos Site)

\section{Discusiones y Resultados}

En los resultados por FRX para la muestra del meteorito, observamos en la Fig. 3 (a) la presencia de los elementos siguientes: $\mathrm{Si}, \mathrm{Cl}, \mathrm{S}, \mathrm{K}, \mathrm{Fe}, \mathrm{Cr}, \mathrm{Cu}, \mathrm{Ca}$, $\mathrm{Ti}, \mathrm{Mn}$ y Ni; con una alta concentración de hierro, así como de azufre, cloro, cromo y níquel en mayores proporciones que en un suelo terrestre (Fig. 3(b) y Tabla 1). En las muestras del suelo del cráter se observan los elementos: $\mathrm{Si}, \mathrm{S}, \mathrm{Cl}, \mathrm{K}, \mathrm{Ca}, \mathrm{Ti}, \mathrm{Mn}, \mathrm{Fe}$, $\mathrm{Ni}, \mathrm{Cu}, \mathrm{Zn}, \mathrm{Rb}$ y $\mathrm{Sr}$; el elemento $\mathrm{Si}$ se halla en mayores concentraciones en comparación con las del meteorito, a diferencia de los elementos $\mathrm{S}, \mathrm{Cr}, \mathrm{Rb}$ y $\mathrm{Ni}$ que se observan en muy bajas concentraciones. La presencia de este último elemento se halla en la siguiente proporción $\mathrm{M} 3>\mathrm{M} 1>\mathrm{M} 2$; el $\mathrm{Ca}$ como $\mathrm{M} 3>\mathrm{M} 2>\mathrm{M} 1$, el $\mathrm{Zn}$ y el Mn como $\mathrm{M} 3>\mathrm{M} 1>\mathrm{M} 2$; el $\mathrm{Cu}$ se observa en proporciones casi invariantes en las tres muestras.

En los resultados por DRX para la muestra del meteorito se observa la presencia de las fases minerales como troilita $(\mathrm{FeS})$, ringwoodita $(\mathrm{Rw},(\mathrm{Mg}$, $\mathrm{Fe})_{2} \mathrm{SiO}_{4}$ ), olivino, y de piroxeno como la fase mineralógica principal (Fig. 4.(a)).

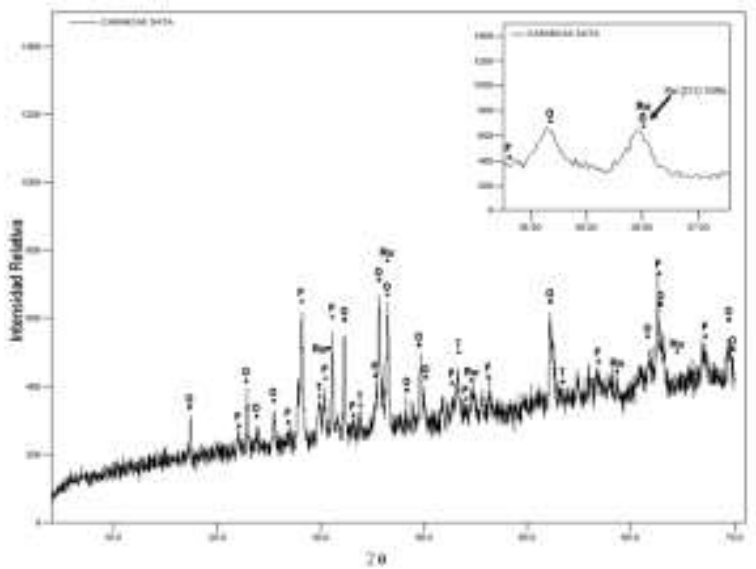

(a)

Fig. 4(a). Difractograma del meteorito de Carancas: Olivino $(\mathrm{O})$, piroxeno $(\mathrm{P})$, troilita $(\mathrm{T})$ y Ringwoodita $(\mathrm{Rw})$. 
En la Tabla 2, se presentan el $\mathrm{N}^{\circ}$ de fichas ICDD de los difractogramas de rayos $\mathrm{x}$ de las muestras analizadas.

En la muestra de meteorito, el origen de Rw (un polimorfo de olivino) se puede relacionar con las elevadas presiones que se alcanzan en los impactos a alta velocidad (Langenhorst y Deutsch, 1998) [2]. También es posible generar fundidos mediante impactos meteoríticos de alta velocidad (Grieve y Cintala, 1992) [3]. La presencia de un mineral metastable de alta presión, como es la Rw, indica una cristalización a presiones entre 13 y 23 Gpa (presión máxima durante el paso de la onda de choque) [4].

Tabla 2 Tabla de fichas ICDD de todas las muestras analizadas por difractometría de rayos $\mathrm{x}$.

\begin{tabular}{|c|c|c|c|c|}
\hline MUESTRAS & CARANCAS & M1 & M2 & M3 \\
\hline \multicolumn{5}{|l|}{ Fases } \\
\hline Olivino & {$[75-1556]$} & & & {$[80-940]$} \\
\hline Troilita & [75-2165] & & & \\
\hline Pyroxeno & [80-276] & & & {$[86-741]$} \\
\hline Ringwoodite & [74-1681] & & & \\
\hline Quartz & & [79-1913] & [82-511] & {$[85-794]$} \\
\hline Albite & & [89-6423] & [83-1606] & {$[84-752]$} \\
\hline Illite & & & [26-911] & [26-911] \\
\hline Montmorillonite & & [13-135] & & [13-135] \\
\hline Hematite & & [88-2359] & [85-599] & \\
\hline Jadeite & & [80-1869] & & [3-635] \\
\hline Coesite & & & [83-1831] & \\
\hline Stishovite & & & [89-3436] & \\
\hline Muscovite & & & [34.175] & \\
\hline Clorita calcica hidratada & & & {$[23-868]$} & \\
\hline Calcite & & & & [47-1743] \\
\hline
\end{tabular}

Este tipo de presiones tan altas son imposibles en la corteza terrestre excepto en el caso de impactos meteoríticos. Bajo esta hipótesis, suponemos que el mineral rico en magnesio precursor de la $\mathrm{Rw}$ (probablemente olivino) previamente habría formado parte del meteorito. Ya que en los meteoritos el olivino se presenta con tres estructuras cristalinas diferentes; por ello, el olivino rómbico es posible que se haya transformado en $\mathrm{Rw}$ y ésto sucede a presiones entre 6 y 16 kbars (según el contenido de $\mathrm{Fe})$ [5]. El mineral primario cuarzo $\left(\mathrm{SiO}_{2}\right)$ y los óxido/óxido-hidróxidos de $\mathrm{Fe}$ no son observados.

A diferencia de estos resultados, todas las muestras del suelo del cráter (Fig. 4 (b), (c) y (d)) revelan una composición que consiste principalmente de cuarzo $\left(\mathrm{SiO}_{2}\right)$ y el silicato albita $\left(\mathrm{NaAlSi}_{3} \mathrm{O}_{8}\right)$.

En la muestra M1 observamos además fases mineralógicas de montmorillonita, hematita y jadeita, siendo ésta ultima un piroxeno; bajo alta presión la albita se descompone para formar la asociación jadeita mas cuarzo. Para las muestras M2 y M3 observamos la presencia de ocho fases primarias, M2 está asociada al cuarzo, illita, albita, hematita, moscovita, clorita calcica hidratada, coesita y stishovita $\left(\mathrm{Si}_{2} \mathrm{O}\right)$. Mientras que en M3 observamos las fases de: cuarzo, albita, illita, montmorillonita, calcita, olivino, piroxeno y jadeita.

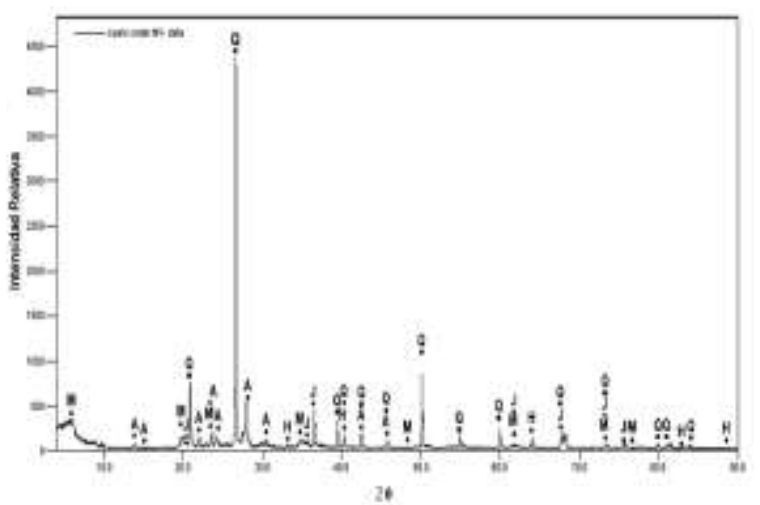

(b)

Fig. 4(b) Difractograma de las muestras M1: Cuarzo (Q), montmorillonita $(\mathrm{M})$, albita $(\mathrm{A})$, hematita $(\mathrm{H})$, jadeita $(\mathrm{J})$ 


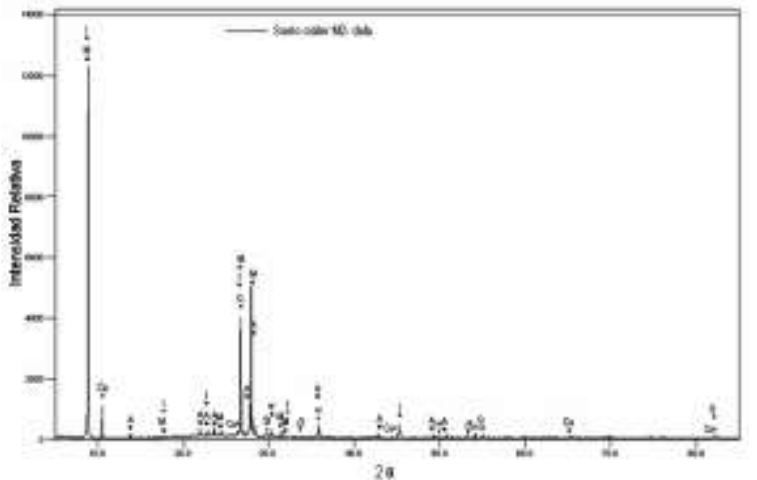

(c)

Fig. 4(c). Difractogramas de la muestra M2: Cuarzo (Q), moscovita (M), albita (A), illita (I), hematita $(\mathrm{H})$, coesita $(\mathrm{Co})$, stishovita $(\mathrm{St})$, clorita cálcica hidratada $(\mathrm{Ca})$.

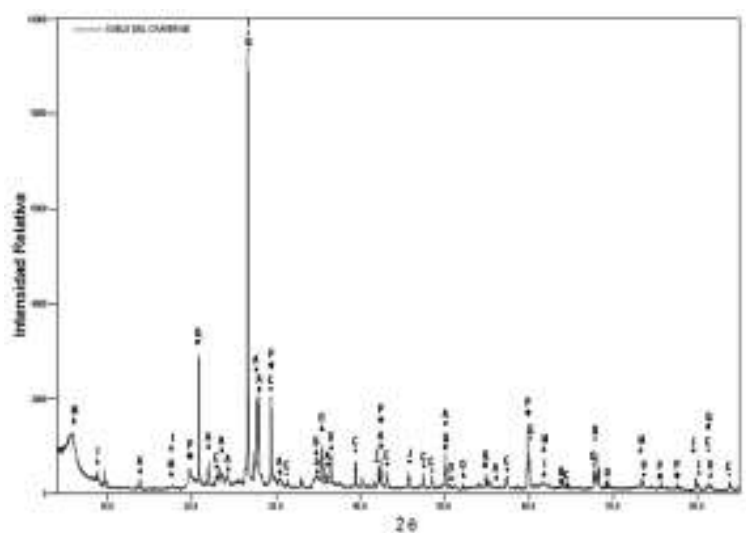

(d)

Fig. 4(d). Difractogramas de la muestra M3: Cuarzo (Q), montmorillonita $(\mathrm{M})$, albita $(\mathrm{A})$, illita $(\mathrm{I})$, calcita $(\mathrm{Ca})$, olivino $(\mathrm{O})$, piroxeno $(\mathrm{P})$, jadeita $(\mathrm{J})$.

El sílice es uno de los componentes más comunes y durables de la corteza terrestre en forma de cuarzo alfa trigonal. Durante un gran impacto meteorítico dos polimorfos más densos que la sílice, la coesita monoclínica y la stishovita tetragonal, pueden formarse a partir de cuarzo a presiones mayores que 3 a $10 \mathrm{GPa}$ (Hemley et al., 1994) [6]. Si bien la coesita puede formarse en condiciones de metamorfismo extremo en las profundidades de la Tierra, éstas no son suficientes para producir stishovita. De tal modo que la formación de ésta en la Tierra, solamente puede darse en los sitios de impactos meteoríticos (e.g., Chao et al., 1962) [7] como consecuencia de las fuertes presiones requeridas.

En la Tabla 3 se pueden observar los parámetros hiperfinos de los diferentes subespectros del meteorito tomados a TA y THL y del suelo del cráter tomado a TA. El espectro Mössbauer de la muestra del meteorito de Carancas tomado a TA y a $11 \mathrm{~mm} / \mathrm{s}$, como se muestra en la Fig. 5 (a), fue ajustado con 2 sextetos magnéticos adjudicados a la troilita (FeS) y a la fase $\mathrm{Fe}-\mathrm{Ni}$; y tres dobletes paramagnéticos, dos de ellos adjudicados al $\mathrm{Fe}^{2+}$, uno asociado al olivino $(\mathrm{Fe}, \mathrm{Mg})_{2} \mathrm{SiO}_{4}$ y el otro al piroxeno $(\mathrm{Mg}, \mathrm{Ca}, \mathrm{Mn}, \mathrm{Fe})$ $\mathrm{Si}_{2} \mathrm{O}_{6}$, y el tercero es debido a un sitio $\mathrm{Fe}^{3+}$ el cual puede estar asociado a óxidos super paramagnéticos y/o hidróxidos de $\mathrm{Fe}$ (en forma de pequeñas partículas de akaganeita, lepidocrocita o goethita) que pueden ser identificados a bajas temperaturas $(4,2 \mathrm{~K})$ [8], como observamos en la Tabla 3.

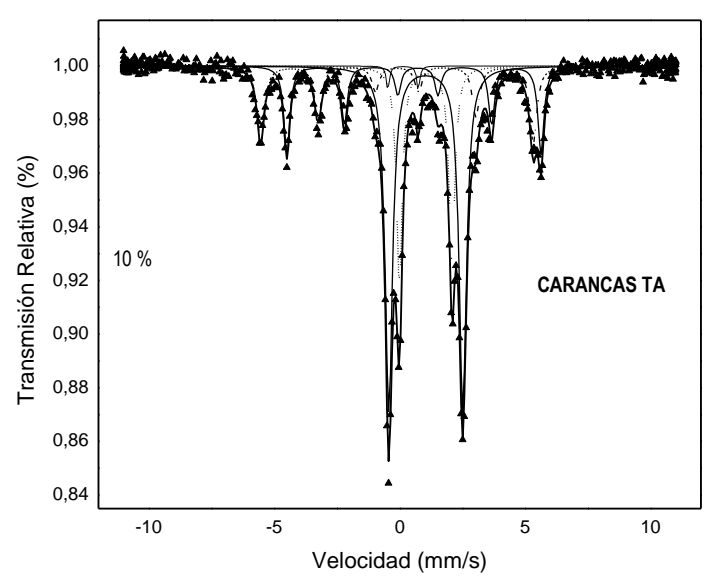

(a)

Fig. 5 (a). Espectros Mössbauer de la muestra del meteorito de Carancas tomado a temperatura de ambiente (TA)

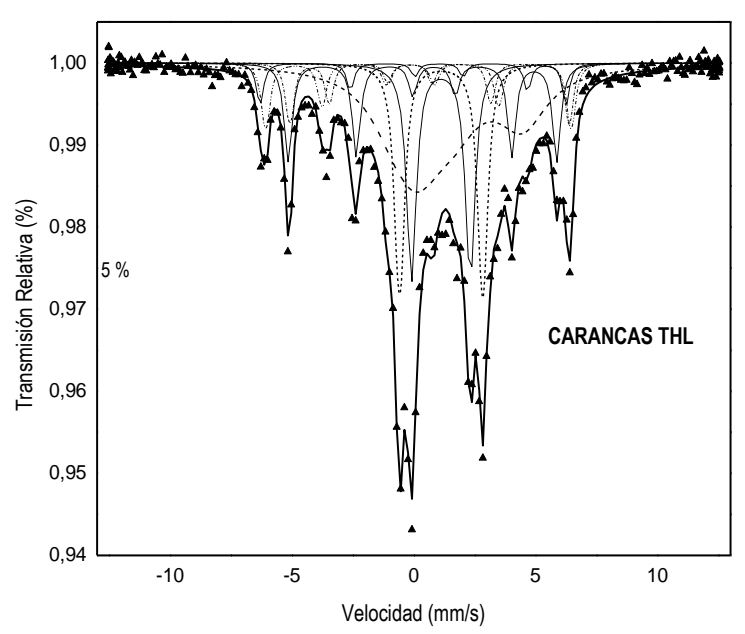

(b)

Fig. 5 (b). Espectros Mössbauer de la muestra del meteorito de Carancas tomado a temperatura de helio líquido (THL).

En los resultados tomados a THL (Fig. 5.(b)), observamos la presencia de cuatro componentes magnéticos ordenados de $\mathrm{Fe}^{3+}$ que están asociados a la akaganeita, a la troilita, y a dos sextetos atribuidos a la fase $\mathrm{Fe}-\mathrm{Ni}$, que podrían asociarse a la taenita 
cristalográficamente ordenada, $\langle\mathrm{Bhf}\rangle=33,55 \mathrm{~T}$, y a la taenita-fase desordenada, $\langle\mathrm{Bhf}\rangle=38.86 \mathrm{~T}$.[9]. El olivino puede aparecer en los meteoritos con tres estructuras cristalinas diferentes. De los resultados observados, es probable que debido a ello se observe el olivino asociado a un doblete paramagnético y a una distribución magnética, $\langle\mathrm{Bhf}\rangle=13,56 \mathrm{~T}$. El piroxeno $\left(\mathrm{Fe}^{2+}\right)$ sigue siendo un doblete paramagnético.

Tabla 3. Parámetros hiperfinos de las muestras del meteorito de Carancas- Puno, y de las muestras M1, M2 y M3 del suelo del cráter.

\begin{tabular}{|c|c|c|c|c|}
\hline \multirow[t]{2}{*}{ MUESTRAS } & \multicolumn{4}{|c|}{ CARANCAS (TA) } \\
\hline & ISO $(\mathrm{mm} / \mathrm{s})$ & QUA (mm/s) & BHF (T) & $\mathrm{A}(\%)$ \\
\hline Fase & -0.110 & 0.000 & 33.670 & 18.450 \\
\hline Troilita & 0.648 & -0.160 & 31.380 & 17.380 \\
\hline Olivino & 1.026 & 2.920 & & 42.030 \\
\hline Pyroxeno & 1.086 & 2.180 & & 20.820 \\
\hline $\mathrm{Fe}^{+3}$ & 0.1270 & 1.013 & & 1.320 \\
\hline \multirow[t]{2}{*}{ MUESTRAS } & \multicolumn{4}{|c|}{ CARANCAS (THL) } \\
\hline & ISO $(\mathrm{mm} / \mathrm{s})$ & QUA $(\mathrm{mm} / \mathrm{s})$ & BHF (T) & $\mathrm{A}(\%)$ \\
\hline Fase FeNi & 0.017 & 0.8235 & 35.55 & 6.790 \\
\hline Fase FeNi & 0.0258 & 0.326 & 38.860 & 7.847 \\
\hline Troilita & 0.586 & -0.458 & 34.162 & 12.566 \\
\hline Olivino I & 1.119 & 3.409 & & 16.007 \\
\hline Olivino II & 1.2425 & 1.8340 & 13.5619 & 38.260 \\
\hline Pyroxeno & 1.116 & 2.380 & & 14.465 \\
\hline Akaganeita & 0.6153 & -0.850 & 38.949 & 4.065 \\
\hline \multirow[t]{2}{*}{ MUESTRA } & \multicolumn{4}{|c|}{ M1-SCRATER } \\
\hline & ISO $(\mathrm{mm} / \mathrm{s})$ & QUA (mm/s) & BHF (T) & $\mathrm{A}(\%)$ \\
\hline $\mathrm{Fe}^{+3}$ & 0.220 & 0.540 & & 54.253 \\
\hline Hematita & 0.2560 & -0.220 & 50.80 & 35.104 \\
\hline Montmorillonita & 1.050 & 2.712 & & 8.947 \\
\hline Illita & 0.590 & 2.180 & & 1.696 \\
\hline \multirow[t]{2}{*}{ MUESTRA } & \multicolumn{4}{|c|}{ M2-SCRATER } \\
\hline & ISO $(\mathrm{mm} / \mathrm{s})$ & QUA $(\mathrm{mm} / \mathrm{s})$ & BHF (T) & $\mathrm{A}(\%)$ \\
\hline $\mathrm{Fe}^{+3}$ & 0.342 & 0.802 & & 35.681 \\
\hline Hematita (I) & 0.256 & -0.20 & 49.20 & 17.575 \\
\hline Hematita (II) & 0.455 & -0.18 & 44.41 & 3.974 \\
\hline Montmorillonita & 1.025 & 1.890 & & 24.908 \\
\hline Illita & 1.000 & 2.306 & & 17.861 \\
\hline \multirow[t]{2}{*}{ MUESTRA } & \multicolumn{4}{|c|}{ M3-SCRATER } \\
\hline & ISO $(\mathrm{mm} / \mathrm{s})$ & QUA (mm/s) & $\mathrm{BHF}(\mathrm{T})$ & $\mathrm{A}(\%)$ \\
\hline $\mathrm{Fe}^{+3}$ & 0.180 & 0.560 & & 54.540 \\
\hline Hematita & 0.256 & -0.220 & 50.57 & 34.966 \\
\hline Montmorillonita & 1.220 & 2.286 & & 9.088 \\
\hline Illita & 0.830 & 1.612 & & 1.406 \\
\hline
\end{tabular}


En la Fig. 6 observamos los espectros de las muestras del suelo del cráter con sextetos magnéticos adjudicados a la presencia de la hematita $\left(\alpha-\mathrm{Fe}_{2} \mathrm{O}_{3}\right)$; y tres dobletes cuadrupolares asociados a sitios de $\mathrm{Fe}$ presentes en la montmorillonita $\left(\mathrm{Fe}^{2+}\right)$ $(\mathrm{Na}, \mathrm{Ca}) 0.33(\mathrm{Al}, \mathrm{Mg})_{2} \mathrm{Si}_{4} \mathrm{O}_{10}(\mathrm{OH})_{2} / \mathrm{nH}_{2} \mathrm{O}$, illita $\left(\mathrm{Fe}^{2+}\right.$, $\left.\mathrm{Fe}^{3+}\right)\left(\mathrm{K}, \mathrm{H}_{2} \mathrm{O}\right) \mathrm{Al}_{2}(\mathrm{Al}, \mathrm{Si}) \mathrm{Si}_{3} \mathrm{O}_{10}(\mathrm{OH})_{2}$ y en una fase de $\mathrm{Fe}^{3+}$.

Podemos observar que la composición elemental obtenida por FRX concuerda con la composición elemental de las fases estructurales halladas por DRX y EMT para la muestra del meteorito como troilita (FeS), olivino ( $\mathrm{Fe}, \mathrm{Mg})_{2} \mathrm{SiO}_{4}$, piroxeno $(\mathrm{Mg}, \mathrm{Ca}, \mathrm{Mn}$, $\mathrm{Fe}) \mathrm{Si}_{2} \mathrm{O}_{6}$ y la fase $(\mathrm{Fe}, \mathrm{Ni})$.

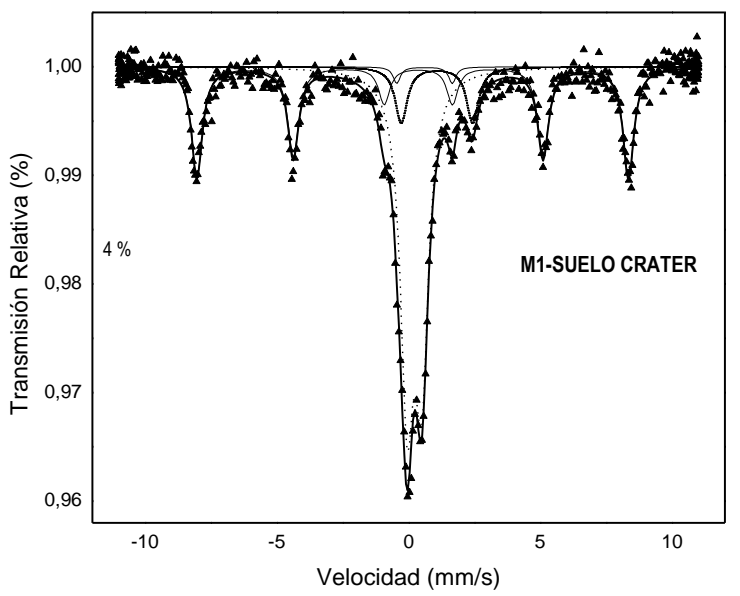

(a)

Fig. 6 (a). Espectro Mössbauer de la muestra M1, tomado a temperatura de ambiente (TA).

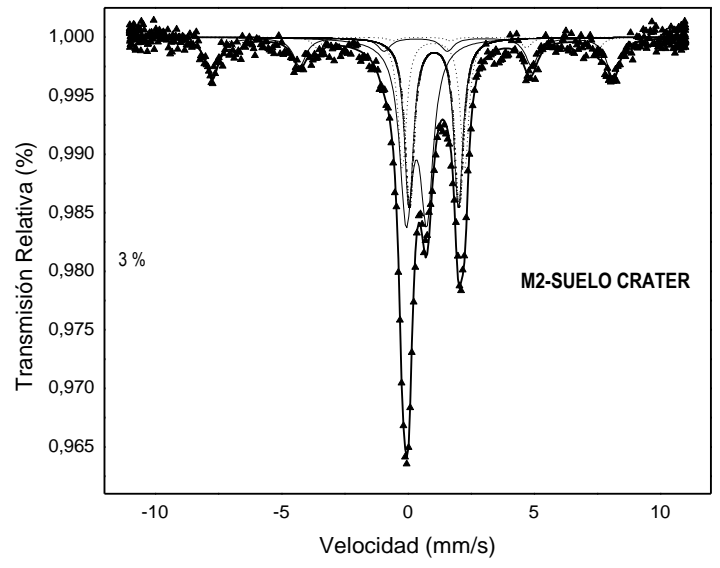

(b)

Fig. 6 (b). Espectro Mössbauer de la muestra M2, tomado a temperatura de ambiente (TA).

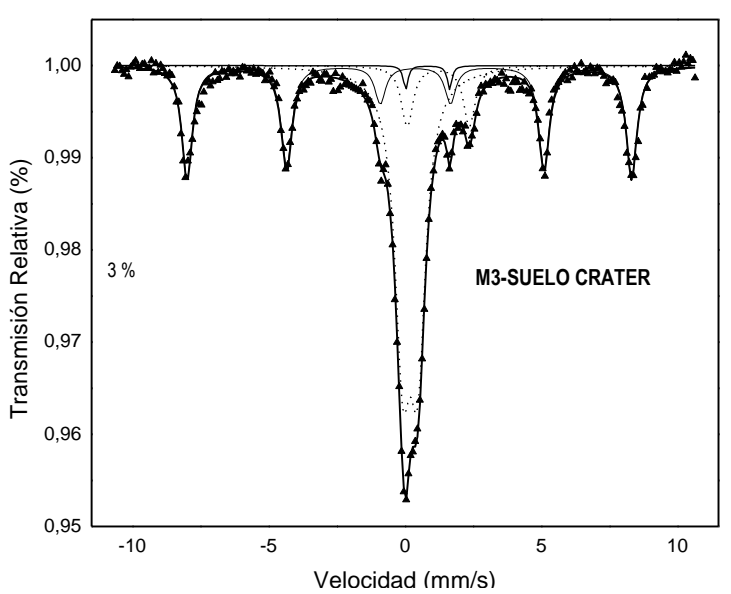

(c)

Fig. 6 (c). Espectro Mössbauer de la muestra M3, tomado a temperatura de ambiente (TA).

\section{Conclusiones}

1.- Los análisis realizados muestran la bondad de las técnicas utilizadas para obtener información básica sobre las muestras estudiadas. Esta información es fundamental para clasificar este meteorito.

2.- Los resultados por FRX permiten diferenciar la composición elemental de las muestras del meteorito de la de una muestra de suelo. Observamos la presencia del $\mathrm{S}$, mineral hallado por esta técnica, el cual fue probablemente responsable del malestar ocasionado a la población de Carancas.

3.- Por DRX, en el caso del meteorito, observamos las fases estructurales del olivino y de la Rw. Esto nos permite confirmar la presencia del mineral $\mathrm{Rw}$ con estructura espinela, con pico principal 100 (311). La presencia de las dos fases, olivino y $\mathrm{Rw}$, indicaría que parte del olivino se transformó en Rw y que la presión del impacto meteorítico habría oscilado entre 6 y 16 kbars; la presencia de Rw es una evidencia de las altas presiones producidas por el impacto meteorítico acaecido en Carancas-Puno.

Por EMT, tomada a TA, observamos fases magnéticas y paramagnéticas solapadas; a THL la fase paramagnética $\mathrm{Fe}^{3+}$ se resuelve como la akaganeita, la fase $\mathrm{Fe}-\mathrm{Ni}$, podría ser kamacita ó taenita (cristalina o desordenada), se sugiere aplicar un campo magnético externo, para resolver mejor esta fase estructural. El olivino presenta ambas fases, paramagnética $y$ magnética.

4.- En todas las muestras estudiadas del suelo del cráter observamos la presencia de la hematita, que se halla en suelos de producción agrícola, y la presencia de impactitas como coesita y stishovita 
para la muestra M2, que son minerales polimorfos de alta presión que hasta ahora sólo han podido ser identificadas en las rocas que contienen cuarzo y han sufrido un metamorfismo de impacto dentro de los cráteres de impacto producidos por meteoritos. Existe solapamiento de picos de diferentes fases, que podría reducirse si utilizamos rayos $\mathrm{X}$ de mayor longitud de onda que la utilizada en este trabajo. Para un mejor resultado de las fases se sugiere hacer el análisis por radiación sincrotrónica, así como también emplear la técnica de espectrometría Raman.

\section{Agradecimiento}

Se agradece el financiamiento del Consejo Superior de Investigación de la Universidad Nacional Mayor de San Marcos a través del contrato $\mathrm{N}^{\circ}$ 081301091. Asimismo, se agradece el apoyo recibido del Centro Brasileño de Pesquisas Físicas del Brasil y de los laboratorios de investigación de la Facultad de Ciencias Físicas de esta Universidad.

\section{6.- References}

[1].Brand, R.A., NORMOS: Mössbauer Fitting Program.

[2].Langenhorst F., y Deutsch, A., (1998). Advanced Mineralogy, vol. 3, Springer-Verlag, p. 95-110.

[3].Grieve, R.A.F., y Cintala, M.J., 1992. Meteoritics
Planet. Sci., 27, 526-538.

[4].Díaz Martínez, E., Sanz Rubio, E., Fernández, C., Martínez Frías, J. (2001a): Evidencia de un pequeño impacto meteorítico en Extremadura: el "volcán" de El Gasco (Las Hurdes). Geogaceta, 30: 47-50.

[5].Melgarejo, J. C. (2004). Atlas de asociaciones minerales en lámina delgada. Vol.1 Editor Joan Charles Melgarejo. España.

[6].Hemley, R.J., et al., (1994). Raman spectrum of natural and sysnthetic stishovite. Physics and chemistry of Minerals, 13, 285-290.

[7].E.C.T. Chao et al., (1962). "First natural ocurrente of coesite", vol. 132, no. 3421, pp. 220222.

[8]. Munayco S. P., Varela E. M., Scorzelli R.B., De Campos J. B., et al. (2008). Room and Low Temperature Mossbauer Spectroscopy for Ordinary Chondrites from The San Juan Strewnfield at the Atacama Desert (Chile). Lunar and Planetary Institute. 71 st The Annual Meeting of the Meteoritical Society. July 28-August 1. MetSoc

[9]. De Grave E., Pollard R. J., Vandenberghe R. E. and De Bakker P. M. A. (1994). The effect of high external magnetic fields on the hyperfine interacctions in the $\mathrm{Fe}-\mathrm{Ni}$ phases of the Santa Catharina meteorite. Hyperfine Interactions 942349- 2353.

[10] Stevens, J. (Editor) (1998). Mössbauer Minerals Handbook, Mössbauer Effect Data Center, Ashville, NC. 